\title{
Qualitative serum organic acid profiles of HIV-infected individuals not on antiretroviral treatment
}

\author{
Aurelia Williams ${ }^{1}$, Gerhard Koekemoer ${ }^{2}$, Zander Lindeque ${ }^{3}$, Carolus Reinecke ${ }^{3}$ \\ and Debra Meyer ${ }^{1 *}$
}

*Author to whom correspondence should be addressed:

Tel: +27 12420 2300; Fax: +27 123625302

debra.meyer@up.ac.za

\begin{abstract}
The first application of gas chromatography mass spectrometry (GC-MS) metabolomics to the analysis of organic acid profiles in sera of asymptomatic HIV-infected individuals $(n=18)$ compared to uninfected controls $(n=21)$, is reported here. Several organic acids are well-established diagnostic biomarkers of mitochondrial dysfunction, making the analysis of the organic acid metabolome well suited to monitoring the progressive disruption of mitochondrial structure and function during HIV infection. Using a multifaceted analyticalbioinformatics procedure, at least 10 of these metabolites could be linked to (1) disrupted mitochondrial metabolism, (2) changes in lipid metabolism and (3) oxidative stress, all of which are aberrations caused by HIV infection. Because of the role of the mitochondria in apoptosis, higher levels of this type of cell death in infected (compared to uninfected) individuals was used to support GC-MS data. This study demonstrates that mass spectrometry metabolomics detects biomarkers of mitochondrial dysfunction which could potentially be developed into indicators of HIV infection, perhaps also to monitor disease progression and the response to antiretroviral treatment (ART).
\end{abstract}

\footnotetext{
${ }^{1}$ Biochemistry Department, University of Pretoria, Pretoria 0002, South Africa

${ }^{2}$ Statistical Consultation Service, North-West University (Potchefstroom Campus), Potchefstroom, 2520, South Africa

${ }^{3}$ Centre for Human Metabonomics, North-West University (Potchefstroom Campus), Potchefstroom, 2520, South Africa
} 
Keywords: Asymptomatic HIV • GC-MS • Multivariate statistics •

- Organic acids • Mitochondrial dysfunction $\bullet$ Metabolomics $\bullet$

\section{Introduction}

The acquired immunodeficiency syndrome (AIDS) is the last of four clinical stages in individuals infected by the human immunodeficiency virus (HIV, World Health Organization 2005; Centlivre et al. 2007). A wide range of immunological, protein and macromolecular markers are identified as characteristic of disease progression (Calabrese et al. 1984 and recently reviewed by Kanekar 2010). The advent of proteomic technologies has contributed significantly to an understanding of the general as well as specific pathological consequences of HIV infection, such as neuroAIDS (Pendyala and Fox 2010). The work by Pendyala and Fox (2010) is a good recent example of "omics" technology applied to HIV research. Metabolomics investigations based on HIV infection are limited. This is apparent from the absence of any reference to low molecular weight molecules in Kanekar's (2010) review on biomarkers of HIV infection.

There are several prognostic markers for HIV infection; CD4 cell count, viral load and to a lesser extent serum neopterin and ßeta-2 microglobulin levels (Touloumi and Hatzakis 2000). Of these examples, only the CD4 count and viral load are used clinically. CD4 counts vary based on blood collection time, other infections, general well-being etc, making it an unreliable marker for disease prognosis (Touloumi and Hatzakis 2000). Metabolic parameters are presently being investigated for markers of infection in part because hypermetabolism was recognized early on as a characteristic of the second and asymptomatic stage of HIV infection (Hommes et al. 1990). These analyses are primarily being done through conventional assays (Hattingh et al. 2009) which are subject to interferences, is laborious to perform, and in addition lack sensitivity and specificity. Changes in body composition, as indicators of metabolic parameters, are recorded using dual energy X-ray absorptiometry, computer tomography scanning and magnetic resonance imaging which are expensive, cumbersome to execute (Gkrania-Klotsas and Klotsas 2007) and require expert interpretation (Wohl et al. 2006). Metabolomics offers several advantages over convention. Firstly, the metabolic profiles can be obtained noninvasively, are characteristic of the individual's phenotype and therefore associated with less variation. Collecting data is fast and high-throughput analysis is possible. Metabolomics techniques are sensitive (Wikoff 
et al. 2008) and allow a range of molecules to be profiled at once, in keeping with the complex and interconnected nature of metabolic pathways.

In a preliminary study, Meyer and co-workers (Hewer et al. 2006) have shown that metabolomics data contain sufficient information to distinguish HIV-positive/AIDS patients on ART from uninfected individuals. This finding was based on metabolite profiles of serum determined by ${ }^{1} \mathrm{H}$ nuclear magnetic resonance (NMR) spectroscopy and linear discriminant analysis. These results were substantiated by a subsequent metabonomics investigation by the same group using 300- and 600-MHz NMR instruments and multinomial logistic regression classification of the metabonomics data (Philippeos et al. 2009). The chemical compounds corresponding to the sections of the NMR spectra that showed significant differences $(P<0.05)$ for infected and uninfected individuals were mainly lipids, including low-density lipoprotein (LDL) and very low-density lipoprotein (VLDL). These observations concurred with the irregularities of lipodystrophy and hyperlipidaemia common in HIV/AIDS patients on ART (Calza et al. 2003). Metabolic changes induced by HIV and/or ART can therefore be revealed by NMR metabonomics.

Wikoff et al. (2008) described changes in the cerebrospinal fluid (CSF) metabolome of rhesus macaques infected with simian immunodeficiency virus (SIV), using GC-MS. In the non-human primate model, Wikoff et al. (2008) used capillary reverse-phase chromatography interfaced with a time-of-flight mass spectrometer (TOF-MS) for a global metabolomics approach to examine the CSF from 4 rhesus macaques before infection with SIV, late in infection, and at sacrifice. The findings obtained clearly indicated that distinct changes in the metabolome were observed as a consequence of SIV-induced encephalitis. Wikoff et al. (2008) concluded that the metabolites identified did not share any structural or chemical characteristics that could be related to a single biochemical mechanism underlying their increase. In addition to the global approach (i.e. untargeted analysis of the metabolome) followed by Wikoff et al. (2008), metabolomics investigations may also benefit from focusing on a specific section of the metabolome as presented here.

It is an established fact that HIV acts directly on the regulation of pathways associated with apoptosis (recently reviewed by Pinti et al. 2010), and that the mitochondria play a central role in this type of cell death. Metabolites that reflect mitochondrial (dys) function could thus represent that section of the metabolome important in a directed metabolomics study.

HIV-induced mitochondrial dysfunction is commonly measured by monitoring apoptosis. Using flow cytometry, Macho et al. (1995) confirmed that mitochondrial dysfunction was evident in the T cells of HIV- 
infected individuals after recording a decrease in mitochondrial membrane potential and a rise in superoxide anion production in these cells. Although flow cytometry is used to measure metabolic changes, it is inadequate for analysing a cascade or profile of metabolites produced by enzymatic reactions (Whitmore et al. 2007; Krylov et al. 2000). Organic acids are generally agreed to be biochemical indicators, or diagnostic biomarkers, of mitochondrial dysfunction (Hoffman and Feyh 2005). Many of these organic acids are highly specific markers used to detect neonatal inborn errors of metabolism (Pitt et al. 2002). Organic acid analysis also proved valuable in our recent study that revealed a global profile of perturbations related to disorders of mitochondrial components (Reinecke et al. 2011). These few examples indicate that organic acids are appropriate components of the metabolome for investigating the possible metabolic effects of HIV infection.

Our initial study on HIV (Hewer et al. 2006) is referred to in an overview by Nordström and Lewensohn (2010) as an example of a metabolomics investigation with potential to provide biomarkers for specific diseases. Here we present an extension of our NMR-based investigations, in which GC-MS was used to analyse the sera from HIV+ individuals. The subjects selected for this study were HIV+, at the second clinical stage of infection, and not on ART. These subjects were selected to determine whether GC-MS metabolomics data could detect metabolic perturbations during this stage of the disease, and whether predictors of disease progression could be detected prior to the development of clinical symptoms of HIV infection. Using a multifaceted analytical-bioinformatics procedure, the identification of statistically significant indicators of the metabolic profile in the second stage of HIV infection was made. Whereas the metabolomics investigation of SIV-infected rhesus macaques revealed distinct differences in the CSF metabolome (Wikoff et al. 2008), the results reported here are the first for treatment-naive HIV+ humans. Conclusions presented pave the way for subsequent investigations, using larger cohorts of participants, to evaluate the practical value of metabolic indicators of HIV infection, disease progression and eventually the response to treatment.

\section{Materials and methods}

\section{Ethics approval}

Ethics approval for the use of human blood in this research was obtained from the Faculties of Natural and Agricultural Sciences and Health Sciences of the University of Pretoria with protocol numbers E080-506019 and 163/2008, respectively. Blood was collected from HIV-seropositive (HIV+) and HIV-negative (HIV-) donors following written informed consent. 


\section{Experimental design}

We selected the organic acid component of the metabolome for this investigation, as explained earlier and next considered which biofluid to use in the analysis. Apoptosis was measured in the peripheral blood mononuclear cells (PBMCs) whereas the organic acid profile was derived from blood serum. Serum was chosen because of its homeostatic regulation and close association with the cells commonly infected by HIV. By measuring mitochondrial dysfunction through GC-MS profiling of organic acids, the profile of these molecules in HIV-infected blood could be obtained. Through this analyses consequences other than the commonly reported immunodeficiency status (low CD4 counts) will be recorded, metabolic changes in the absence of ART determined and the application of GC-MS metabolomics to the study of HIV-infected biofluids evaluated. Blood samples were collected in batches. Batch 1 comprised of $11 \mathrm{HIV}$ - and $12 \mathrm{HIV}+$ samples and batch 2 consisted of $10 \mathrm{HIV}$ - and $6 \mathrm{HIV}+$ samples. Figure 1 gives the sequence of experimental and analytical steps followed from the point of sample collection to the interpretation of the information gained through data analysis.

\section{Sample selection and collection}

Blood samples of HIV+ donors (total $\mathrm{n}=18$ ) were obtained from the Steve Biko Academic Hospital and from King's Hope Development Foundation Clinic in Diepsloot, Johannesburg. The participating donors were recruited from a cohort of individuals who were aware of their HIV-positive status, had not been diagnosed as having AIDS (CD4 counts were above 200 cells $/ \mathrm{mm}^{3}$ blood) and were not on ART. For one of the donors, a CD4 count of less than 200 cells $/ \mathrm{mm}^{3}$ was detected after the start of the study. This case was retained in the experimental group because the individual had not commenced ART. Viral load, measured by the COBAS AmpliPrep/COBAS TaqMan HIV-1 Test (Roche Molecular Systems, Pleasanton, CA) was not part of the inclusion criteria because practices at the source clinics were such that the viral load of individuals was determined only when they were tested for HIV infection for the first time and then immediately before treatment was initiated. Table 1 summarises general and clinical information about the participants. Healthy control donors (total $n=21$ ) were recruited at the campus of the University of Pretoria and had no known metabolic or other medical condition at the time of blood collection. The HIV negative status of these samples was confirmed with VISITECT ${ }^{\circledR}$ HIV $1 / 2$ rapid tests (Omega Diagnostics Limited, Scotland, UK). Immunophenotyping experiments also confirmed that the healthy controls had a significantly higher percentage of CD4 cells than their HIV+ counterparts, which was to be expected because of their HIV-negative status. The negative controls and HIV+ samples used were fairly well-matched in terms of gender (HIV- and HIV+ groups 
BATCH 1

HIV+: 12 Controls: 11

(Clinical detail: Table 1)
BATCH 2

HIV+: 6 Controls: 10

(Clinical detail: Table 1)

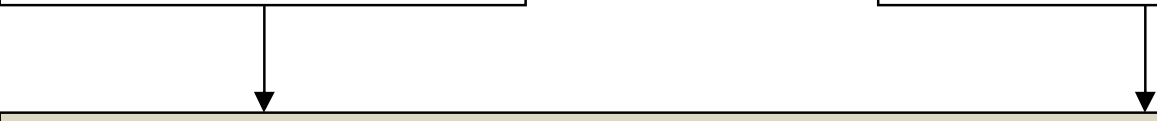

ANALYTICAL PROCEDURES FOR THE IDENTIFICATION OF VARIABLES

1. Denaturation of serum proteins and extraction of hydrophobic substances

2. Derivatization of dried residues

3. GC-MS analyses

4. Peak deconvolution and alignment: AMDIS and MET-IDEA

5. Feature elimination through manual curation

6. NIST: Identify compounds from MET-IDEA data (Retention time and $m z$-values)

7. Selection of endogenous and natural organic acids (HMDB, PubChem, etc.)

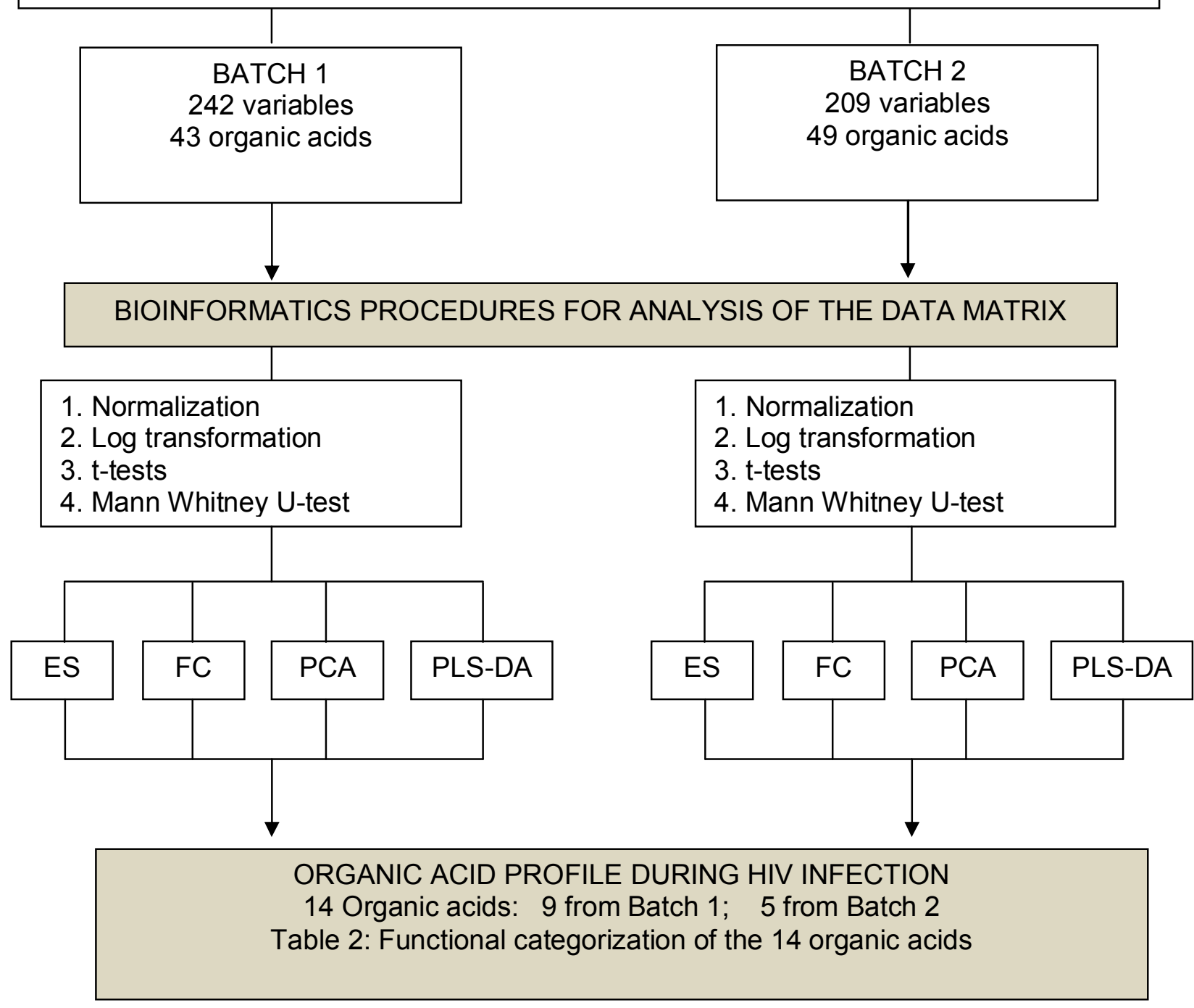

Fig. 1 Schematic representation of the work flow indicating the selection of the experimental groups (HIV- and HIV+) as well as the analytical procedures and bioinformatics used to identify perturbation markers on HIV infection 
totalled $90.9(100 \%)$ females for batch 1, and $60(50 \%)$ females for batch 2) and age (HIV- and HIV+ donors in batch 1 had a mean age \pm SD of $31.91 \pm 12.62$ and $33.42 \pm 7.86$ years, respectively, whereas the corresponding ages for batch 2 were $25.9 \pm 7.29$ and $34.8 \pm 8.93$ years).

\section{Sample preparation}

\section{PBMC isolation}

Venous blood was collected in EDTA vacutainers (Greiner Bio-One, Kremsmünster, Germany) from donors on several occasions. PBMCs were isolated from whole blood by density gradient centrifugation $(1912 \times$ g for 30 minutes) using Ficoll-hypaque (Sigma Chemical Company, St. Louis, MO).

\section{Apoptosis determination}

Apoptosis in HIV- and HIV+ PBMCs was determined by flow cytometry using the annexin $\mathrm{V}$ and propidium iodide (PI) kit (BD Biosciences, California, USA). Cells which stained negative for annexin V-FITC and PI when using the kit were considered to be viable whereas cells that stained positive for annexin V-FITC were apoptotic. Those cells that stained positive for both annexin V-FITC and PI and PI only represented damaged and dead cells respectively (van Engeland et al. 1998). Briefly, $2 \mathrm{ml}$ of cells at $1 \times 10^{6}$ cells $/ \mathrm{ml}$ was washed with cold phosphate buffered saline (PBS, $500 \times \mathrm{g}, 5$ minutes) and the cell pellet resuspended in $100 \mu \mathrm{l}$ binding buffer. Cells were stained with $2 \mu \mathrm{l}$ annexin V-FITC and $2 \mu \mathrm{l}$ PI solution. Cells and dye were mixed and incubated at $4^{\circ} \mathrm{C}$ in the dark for 15 minutes. Binding buffer $(400 \mu \mathrm{l})$ was then added to the cell-dye mixture. At least 10000 events were collected on a FACSAria (BD Biosciences) within 30 minutes of adding binding buffer. Unstained cells, annexin-positive and PI-positive controls were prepared and used for compensation purposes (mathematical elimination of the spectral overlap between different fluorochromes) as well as quadrant specification. Quadrants are defined through the use of fluorescence minus one (FMO) controls. These are staining controls that include all staining reagents except the one of interest and are used to accurately differentiate between cell populations within a stained sample (Roederer 2001). Apoptosis was ultimately reported as the percentage of cells that stained positive for annexin-V FITC. 


\section{Serum preparation}

Samples were collected in non-EDTA vacutainers (Greiner Bio-One) for serum isolation. The time between sample collection and serum preparation or organic acid extraction was approximately 4 hours. Blood samples were allowed to clot in the non-EDTA vacutainers and serum removed after centrifugation at $1610 \times \mathrm{g}$ for 10 minutes at room temperature (Tuck et al. 2009; Verde et al. 2002). Approximately $1.5 \mathrm{ml}$ serum was obtained from $4 \mathrm{ml}$ of blood using these conditions, of which $1 \mathrm{ml}$ was used for organic acid extraction. The remaining serum was stored as aliquots at $-70{ }^{\circ} \mathrm{C}$ for subsequent determination of their oxidative stress and secreted cytokine profiles.

\section{Organic acid extraction}

Organic acids were isolated from HIV- and HIV+ serum by solvent extraction prior to GC-MS analysis according to a published protocol (Reinecke et al. 2011). All reagents used for organic acid extraction were from Merck (Darmstadt, Germany) unless otherwise stated. Extractions were carried out in Kimax glass tubes from LASEC, South Africa. One hundred microlitres of the internal standard (IS), 3-phenylbutyric acid (SigmaAldrich, St Louis, MO) was added to $1 \mathrm{ml}$ of serum to a final concentration of $52.5 \mathrm{mg} / \mathrm{l}$. The $\mathrm{pH}$ of each sample was adjusted to 1 using $5 \mathrm{~N} \mathrm{HCl}$. Ethyl acetate $(6 \mathrm{ml})$ was added to each sample followed by shaking for 30 minutes. Centrifugation at $1118 \times \mathrm{g}$ for 3 minutes at room temperature facilitated phase separation and the resulting organic phase was transferred into a clean glass tube. Diethyl ether $(3 \mathrm{ml})$ was added to the remaining (lower) aqueous phase and the samples shaken for 10 minutes. Phase separation was once again achieved by centrifugation $(1118 \times \mathrm{g}$ for 3 minutes at room temperature) and the resulting organic phase added to the previous extract. Anhydrous sodium sulphate $\left(\mathrm{Na}_{2} \mathrm{SO}_{4}\right)$ was added to the extracted samples and the mixture vortexed and centrifuged. The extracted organic phase of each sample was transferred to a clean glass tube and evaporated to dryness under nitrogen gas at $40^{\circ} \mathrm{C}$.

\section{GC-MS analysis}

All mass spectrometry was performed at the Centre for Human Metabonomics, North West University, Potchefstroom Campus, according to the protocol established there (Reinecke et al. 2011). The dried residue was derivatized for 45 minutes at $70{ }^{\circ} \mathrm{C}$ using $40 \mu \mathrm{N}$, O-bis (trimethylsilyl) trifluoroacetamide (BSTFA, Supelco Analytical, Bellefonte, USA), $8 \mu 1$ trimethylchlorosilane (TMCS, Sigma-Aldrich) and $8 \mu 1$ pyridine (Sigma-Aldrich). The samples were analysed using an Agilent 7890A/5975B inert XL GC-MSD system 
equipped with a DB-1MS capillary column (30 m x $0.25 \mathrm{~mm}$ x $0.25 \mu \mathrm{m})$. The GC oven temperature programme started at $60{ }^{\circ} \mathrm{C}$ for 2 minutes, increased at $4{ }^{\circ} \mathrm{C} / \mathrm{min}$ to $120{ }^{\circ} \mathrm{C}$, and then at $6{ }^{\circ} \mathrm{C} / \mathrm{min}$ to $285{ }^{\circ} \mathrm{C}$, which was maintained for 2 minutes. Samples $(1 \mu l)$ were injected in splitless mode with the injector temperature set to $280{ }^{\circ} \mathrm{C}$. The carrier gas was helium (17.73 psi) and electron impact (EI) ionization was applied at $70 \mathrm{eV} . \mathrm{MS}$ acquisition was performed in scan mode.

\section{Peak deconvolution, alignment and identification}

Peak deconvolution and alignment were carried out using the Automated Mass Spectral Deconvolution and Identification System (AMDIS, version 2.66) from the National Institute of Standards and Technology (NIST) and a Metabolomics Ion-based Data Extraction Algorithm (MET-IDEA, version 2.04, the Roberts Noble Foundation Inc.). All samples were deconvoluted in AMDIS with the following settings: component width 20; adjacent peak subtraction of 1; and resolution, sensitivity and shape requirements adjusted to the lowest setting. The following ion channels were excluded to limit the extraction of false positive peaks: TIC, 73 and $147 \mathrm{~m} / \mathrm{z}$. The NIST 08 library was used to identify the detected compounds. A representative sample from each batch was selected for targeted data extraction using MET-IDEA. The output from AMDIS, that is, the *. elu and *. fin files of the reference sample, were loaded into MET-IDEA to create an ion list which consisted of a model ion paired with its retention time for each compound detected (Broeckling et al. 2006). Data extraction in METIDEA was performed according to Broeckling et al. (2006) using default values for GC coupled to a quadrupole MS instrument.

\section{Data pre-processing}

Data matrices generated by MET-IDEA were inspected for deconvolution errors by examining the peaks and using the correlation function in MET-IDEA. Deconvolution errors occur when a single compound peak is detected as two separate peaks (Xu et al. 2009). These replicate variables were removed by manual curation and the matrices exported, providing 242 and 209 variables for the two batches, respectively. These variables (corresponding to individual chemical compounds) were classified as endogenous, exogenous, and unclassified (if no biological information about them was available), respectively. Variables were categorised by consulting various electronic sources (such as the Human Metabolome Database, PubChem, Google) and 
articles in the scientific literature. Unclassified and exogenous molecules were subsequently excluded from further analysis, leaving batches 1 and 2 with 43 and 49 endogenous molecules, which were processed further.

\section{Data normalization and pre-treatment}

As part of pre-treatment, data were normalized and transformed to remove non-biological variation among samples and to make the scales of the data sets comparable. Normalization was done through the regression approach (Redestig et al. 2009; Sysi-Aho et al. 2007) whereas data were transformed using a shifted $\log$ function. All pre-processing and subsequent statistical analysis was performed using the statistical computing package R (http://www.r-project.org) and STATISTICA (version 10, StatSoft ${ }^{\circledR}$ ). Statistical analysis was conducted as described below, after the data were centred.

\section{Statistical analysis}

Principal component analysis (PCA, Trygg et al. 2007) and partial least squares discriminant analysis (PLS-DA, Barker and Rayens 2003) were performed as part of multivariate analysis. Because the study was explorative, the PLS-DA model was not validated but used to obtain a ranked list of metabolites expressed as variables important in projection (VIP). The measure of modelling power associated with each variable in the PCA analysis was likewise used to rank the metabolites in order of importance.

Effect sizes (ES), which are based on univariate analysis were calculated to assist with the selection of important molecules using normalized, log transformed data. An ES value not only indicates practical significance but also reveals the magnitude of the effect of a perturbation (Nakagawa and Cuthill 2007). The effect size is defined by the equation: $\mathrm{ES}=\left|\bar{X}_{1}-\bar{X}_{2}\right| / \mathrm{S}_{\max }$, where $\bar{X}_{1}=$ mean of the respective variables of the control group, $\bar{X}_{2}=$ mean of the respective variables of the HIV-infected group, and $\mathrm{S}_{\max }=$ maximum standard deviation of the two groups. Effects were classified as small, medium and large, corresponding to ES values of $0.2,0.5$ and 0.8 , respectively. The greater the effect size the greater the contribution of the molecule to the profile recorded. For this study, highly ranked PCA markers, PLS-DA VIPs as well as molecules with ES $\geq 0.8$ were used to identify the most prominent molecules in the chemical profiles of patients and controls. Metabolite concentrations that changed due to HIV infection were identified by fold changes (FC), which were regarded as significant for values $\geq 1.5$ (Tusher et al. 2001). To discover any significant differences between the means and medians of HIV- and HIV+ groups, P values were calculated using t-tests and non-parametric Mann-Whitney tests. 


\section{Results and discussion}

\section{Profile of the experimental groups}

Metabolic fingerprinting of GC-MS data requires pre-processing to generate a data matrix of variables and cases of an operational size, appropriate for multivariate analysis to identify only information relevant to the study. The experimental and analytical steps taken to reach this goal are shown schematically in Figure 1. They consist of three successive phases: (1) selection of the experimental groups; (2) the analytical protocol to derive the metabolomics matrix and (3) the bioinformatics analysis to identify significant indicators of HIV infection.

Both sample groups (21 uninfected, 18 infected) were assigned to two batches, for which the corresponding clinical information is summarized in Table 1. More women than men were involved in this study, which reflects the HIV infection pattern reported for sub-Saharan Africa, where HIV prevalence is higher in women (UNAIDS Report 2002 and 2009). The mean CD4 counts of the HIV+ individuals for the two batches were 377 (SD 209) and 342 (SD 64) cells per $\mathrm{mm}^{3}$ blood, respectively (Table 1). Within batch 1, two individuals presented with CD4 counts above 600 , and one with a value below 200 cells per $\mathrm{mm}^{3}$ blood. Given the limited number of participants, these three cases were retained in subsequent analyses as they complied with the three primary requirements for inclusion as patients in this investigation, namely; they were HIV+, asymptomatic and not on ART. The majority of the HIV+ cases were within the category for which treatment is not needed (> 350 cells per $\mathrm{mm}^{3}$ blood, World Health Organization 2006). According to the WHO guidelines, the individual with the CD count below 200 cells per $\mathrm{mm}^{3}$ blood should already have started ART, irrespective of clinical status, which is what we recommended.

The range of the viral load in batch 1 was $78000-952000$ copies / ml plasma (mean = 382000$)$ and $2300-178000$ in batch 2 (mean 73 000). The mean viral load for batch 1 was therefore affected by the inclusion of the individual advancing to AIDS. These values can be interpreted only qualitatively, but suggest that the disease had probably progressed to a somewhat more advanced stage for those individuals in batch 1 than those in batch 2 (Jansen et al. 2006). Although the mean age of the HIV+ individuals in the two groups were comparable, we decided not to combine the batches but to analyse them separately, as shown in Figure 1 .

\section{Apoptosis Profile}

An indication of mitochondrial function can be obtained by measuring the apoptosis profile of immune cells. An example of the experimental results indicating apoptosis is shown in Figure 2. Apart from one outlier 

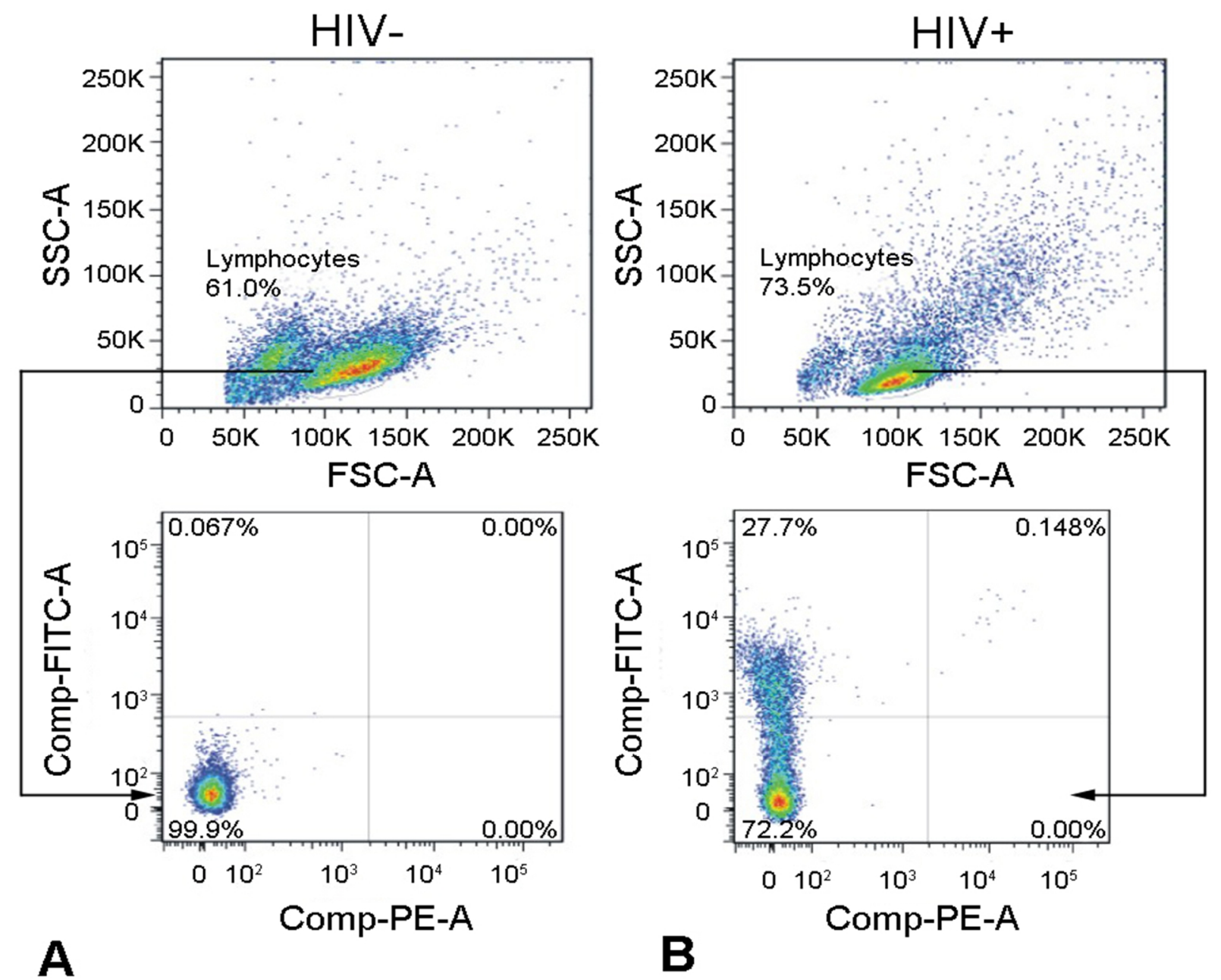

Fig. 2 Gating strategy used for determining apoptosis in PBMCs. Lymphocytes was selected using side scatter (SSC) and forward scatter (FSC) light properties. Within the lymphocyte gate the $\%$ apoptotic PBMCs positive for annexin-V was measured in the FITC channel. Damaged, dead cells positive for PI were detected in the PE channel. Shown here are representative examples of apoptosis determinations in a) HIV- and b) HIV+ cells respectively 


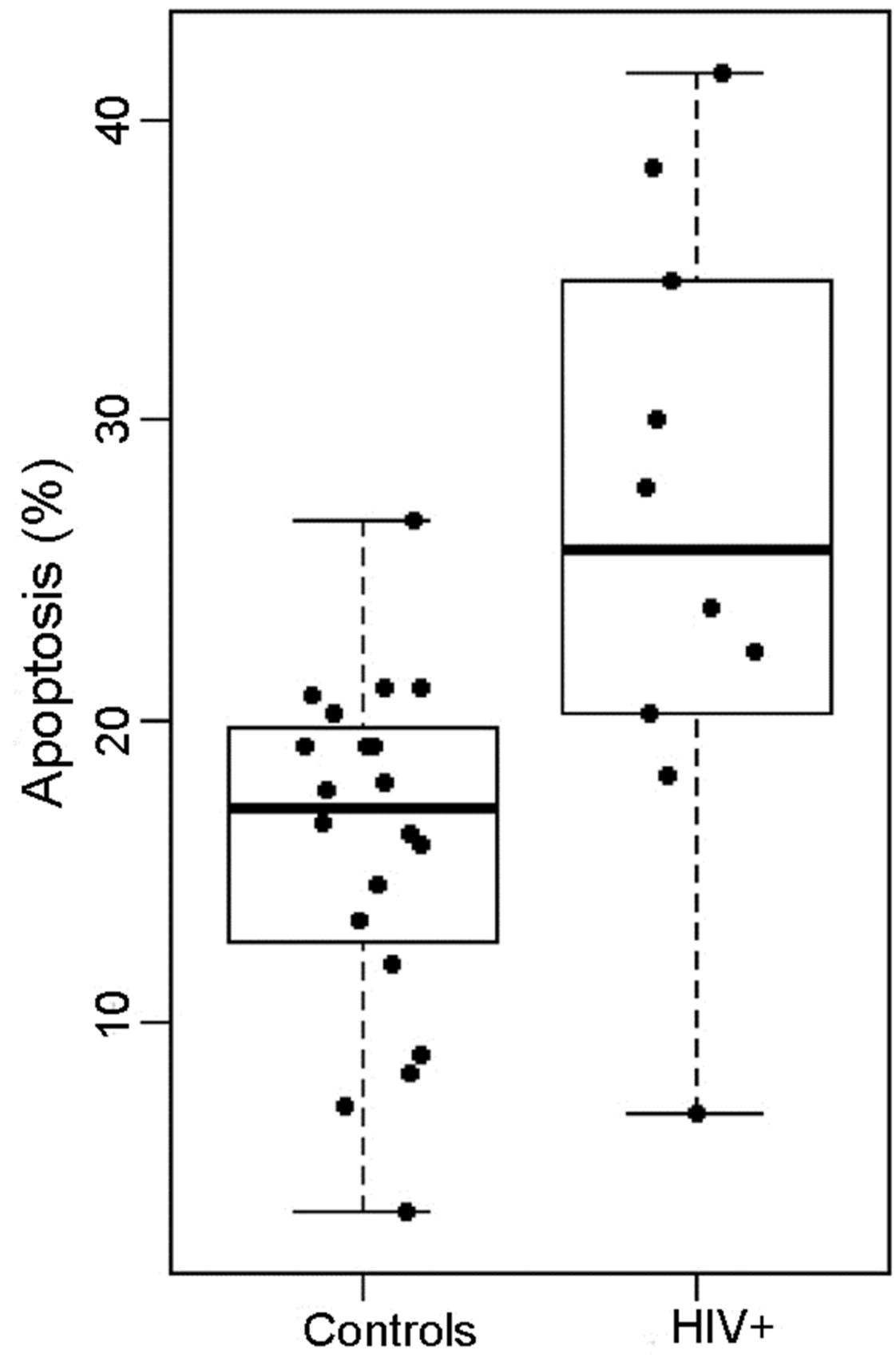

Fig. 3 Apoptosis profile of the uninfected controls and HIV+ individuals. The percentage apoptosis in HIV+ individuals was significantly higher $(\mathrm{p}=0.012$ (t-test) and $\mathrm{p}=0.004$ (Mann-Whitnety U-test)) than in controls 
corresponding to a low level of apoptosis, Figure 3 indicates a significant $(p=0.012$ (t-test) and $p=0.004$ (Mann-Whitnety U-test)) increase in apoptosis as a characteristic of the HIV+ cells relative to the uninfected controls. This concurs with apoptosis being one of the main mechanisms of CD4 depletion during HIV infection (Banda et al. 1992; Meyaard et al. 1992) and inform on the profile of apoptosis in the patient group used in this investigation. The level of HIV-induced apoptosis thus exceeds that of normal programmed cell death in healthy individuals (Roshal et al. 2001).

\section{GC-MS data and analytical procedures for the identification of variables}

Data generation and pre-treatment for the two batches (each comprising controls and HIV+ individuals) were conducted separately. The same operational procedures were used in both instances, as shown in Figure 1. The isolation of the hydrophobic substances, derivatization and GC-MS analysis was carried out according to the protocol standardized in our laboratory (Reinecke et al. 2011). A customized AMDIS software package was used for data extraction and peak deconvolution. This yielded 282 features for batch 1 and 237 for batch 2 . These compounds correspond closely with the 300 organic acids or related compounds believed to be among the total set of 4229 compounds recently reported for the human serum metabolome (Psychogios et al. 2011).

The AMDIS data included some typical noise and contaminating ions. A supplementary list of ionretention time pairs was generated to guide a selective ion-based data extraction process using MET-IDEA. The list still contained a few duplicate features (Dunn et al. 2011) that were removed by manual curation using the AMDIS deconvolution and MET-IDEA alignment information. This procedure reduced the number of features for batch 1 to 242 and for batch 2 to 209 . These numbers are considerably smaller than the 3687 compounds identified in the global metabolomics analysis of CSF from SIV+ rhesus macaques (Wikoff et al. 2008), but in line with the selective metabolome analysis presented here. The NIST library was subsequently used to assign names to more than $90 \%$ of the compounds extracted from both batches 1 and 2 . The remaining $10 \%$ were regarded as unknown (unidentifiable or not possible to annotate) and not considered in the subsequent analyses. Using public databases, those compounds known to be endogenous human organic acid metabolites were selected from the two lists of compounds identified from batches 1 and 2 . This resulted in 43 organic acids assigned to batch 1 and 49 to batch 2 . The final outcome of the analytical protocol was thus matrices for batch 1 and 2, each comprising controls and HIV+ cases, and a list of known compounds (variables/metabolites) expressed as numerical values for the respective model ions. These matrices were regarded as suitable for identifying indicators/markers that were differentially present in the HIV+ individuals and uninfected controls. 


\section{Bioinformatics procedures for analysis of the data matrix}

The intensity values of the extracted ions (variables) from the MET-IDEA analysis were firstly used in the fold change analysis. The fold changes for the 40 organic acids which increased in batch 1 ranged from 1.07 to 38.65 , of which $34(79.1 \%)$ had fold changes $\geq 1.5$. For batch 2 the respective increases ranged from 1.01 to 61.84 for 44 organic acids, and $37(75.5 \%)$ had fold changes $\geq 1.5$. Only a limited number of organic acids decreased due to HIV infection: these were $3(7.0 \%)$ for batch 1 (range of fold change: 1.12 to 1.65 ), and 5 (10.2\%) for batch 2 (range of fold change: 1.11 to 12.21 ). These results clearly indicate the potential value of organic acids as biomarkers for HIV infection. The fold changes found for the indiviual organic acids were thus taken into account together with other statistical information for the selection of important metabolite markers, as will be discussed below. Secondly the intensity values from the MET-IDEA analysis was normalized and log transformed prior to applying statistical methods to identify important markers. A scatter plot of the mean log (relative intensity) of all identified metabolites for batches 1 and 2 was generated and is shown in Figure 4. It is clear that most of the identifiable organic acids increased in relative intensity in the HIV+ cases compared to the controls, in agreement with the observed fold changes for the unscaled values. A similar observation was made by Wikoff et al. (2008) for features which had a $P$ value less than or equal to 0.01 , indicating that most of the metabolite concentrations increased significantly during SIV-induced encephalitis as well.

Univariate (effect size) analysis was subsequently performed and two multivariate analyses (PCA and PLSDA) conducted to further refine the selection of HIV perturbation markers. Thus, three criteria were used to assess the data of each batch to select these important indicators:

- The effect size of all selected metabolites had to be greater than 0.8 within their batch, to ensure their practical as well as statistical significance.

- All metabolites with ES $>0.8$ from the upper $50 \%$ of the list of metabolites were ranked by the modelling power obtained by PCA.

- All metabolites with ES $>0.8$ from the upper 50\% of the list of VIPs were identified by PLS-DA.

The outcome of the multivariate analyses is shown in Figure 5. The unsupervised PCA indicated sufficient variation between the experimental groups from batch 1 to achieve a complete separation between data from controls and HIV-individuals (Figure 5, left panel). Such a separation was not observed for batch 2. These observations suggest the possibility that batch 1 individuals were at a more advanced stage of disease than those 


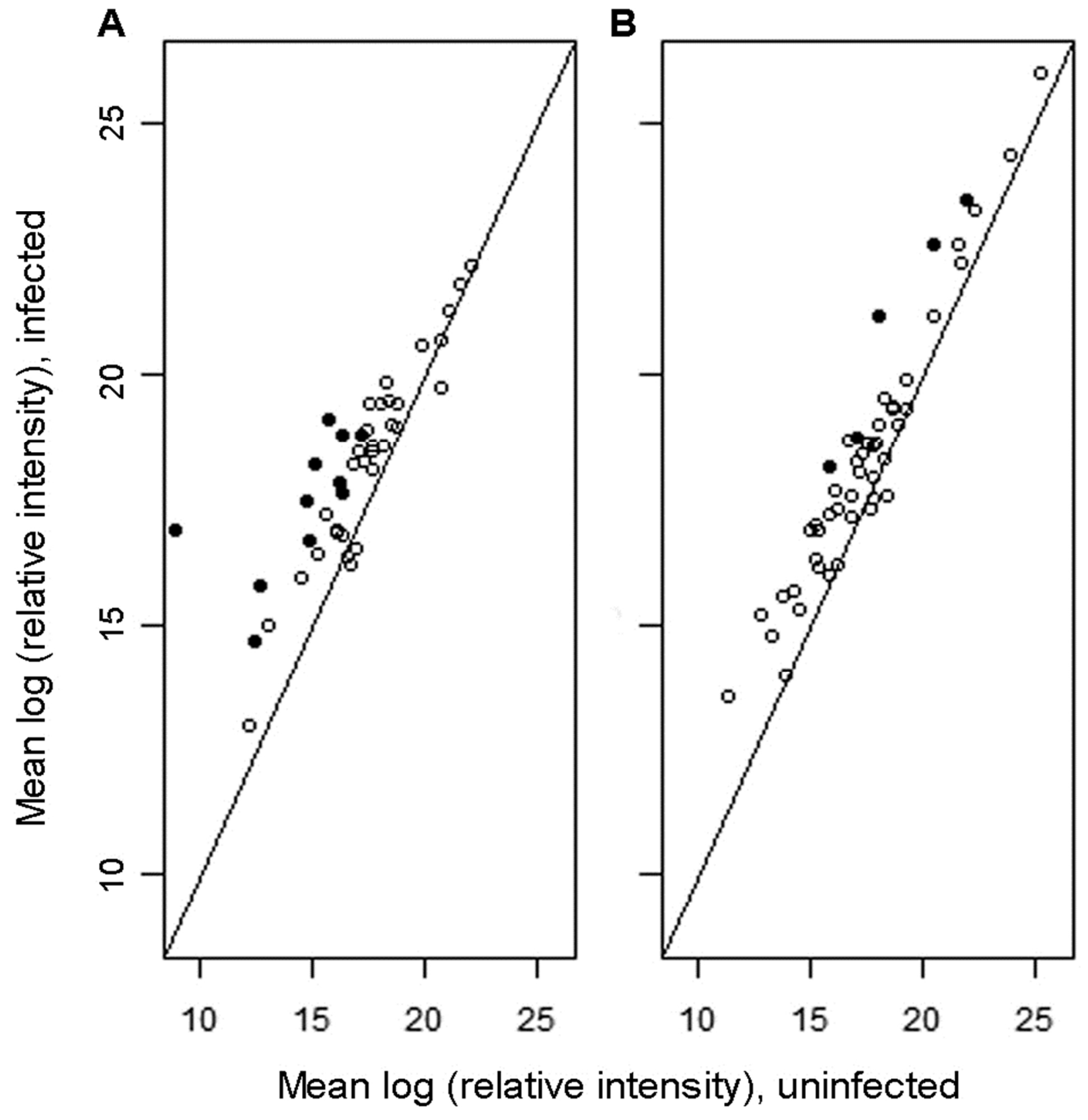

Fig. 4 Scatter plot comparing the integrated intensities of features representing endogenous metabolites in samples from uninfected versus HIV-infected individuals for batch 1 (a) and batch 2 (b). The diagonal line $(y=x)$ serves to indicate which metabolites were increased (left section of the respective figures) in samples from the HIV+ individuals. Filled circles are the position of variables common to the respective PCA and PLS-DA analysis, having effect sizes larger than 0.8 . The open circles are the remaining metabolites from the original 43 and 49 identified from the data for batches 1 and 2 respectively 
Table 1. Meta data of the negative control and HIV + groups in each of the respective batches ${ }^{\mathrm{a}}$.

\begin{tabular}{lll} 
& \multicolumn{2}{c}{ HIV Status } \\
\cline { 2 - 3 } Batch 1 & HIV- & HIV + \\
\hline \% Females & 90.91 & 100.00 \\
\% Males & 9.09 & 0.00 \\
Mean Age \pm SD (years) & $31.91 \pm 12.62$ & $33.42 \pm 7.86$ \\
Mean CD4 count (cells $/ \mathrm{mm}^{3}$ blood) & Not Determined & 376.45 \\
Viral Load (copies $/ \mathrm{ml}$, range) & Not Applicable & $381542(77641-951995)$ \\
\hline & & \\
& & HIV Status \\
\cline { 2 - 3 } Batch 2 & HIV- & HIV+ \\
\hline \% Females & 60.00 & 50.00 \\
Mean Age \pm SD (years) & 40.00 & 50.00 \\
Mean CD4 count (cells $/ \mathrm{mm}^{3}$ blood) & $25.9 \pm 7.29$ & $34.8 \pm 8.93$ \\
Viral Load (copies $/ \mathrm{ml}$, range) & Not Determined & 342.20 \\
\hline
\end{tabular}

\footnotetext{
${ }^{a}$ The clinical data shows that the CD4 counts of the two groups were comparable whereas the viral load was not.
} 
represented by batch 2 (this is also supported by viremia data, Table 1). For each of the batches a list of variables (representing compounds) and their respective modelling power were used to identify important variables contributing to the organic acid profiles of the experimental groups. The results in Figure $5 \mathrm{~b}$ show that complete separation of the controls and HIV+ cases was obtained in the supervised PLS-DA. As with PCA, the lists of variables important in projection were obtained from both batches of sample for which PLS-DA analyses was performed.

All variables in the first half of the lists of variables from the PCA and PLS-DA, having an ES value greater than 0.8 were selected. From this analysis, 19 metabolites were common to the three methods of selection described above. In addition, a fold change for all these variables was used as an additional criterion to confirm that the selected metabolites changed significantly (fold change $\geq 1.5$ ) due to the HIV infection. Subsequently, the standard t-tests and the non-parametric Mann-Whitney U-tests were used to test the equality of group means and group medians, respectively. Only those metabolites that (1) showed statistically significant values from these analyses and (2) are present in the human serum metabolome were finally taken into account to characterize the biological profile of the untreated chronic phase of HIV progression in terms of organic acids and mitochondrial function. Fourteen metabolites with fold change values ranging from 2.95 to 23.62 for batch 1 and from 3.80 to 61.84 for batch 2, complied with all these criteria and are listed in Table 2, confirming also the observations and conclusions made on the scatter plots (Figure 4). The table also shows analytical information (feature names, chemical names and molecular formulae) and statistical information for each metabolite. Representative spectra of two of the identified organic acids (one from each batch) are shown in Figure 6 and compliments the spectra contained in the NIST library (shown as an insert).

\section{Metabolic interpretations of the markers of HIV infection}

The functional and other information revealed by the metabolites summarized in Table 2 can be classified into four categories:

- Markers of mitochondrial/metabolic dysfunction

- Saturated and unsaturated fatty acids, other lipids and metabolites involved in lipid metabolism

- Oxidative stress-related metabolites

- Other metabolites recognized as part of the human serum metabolome

These are elaborated on below. 


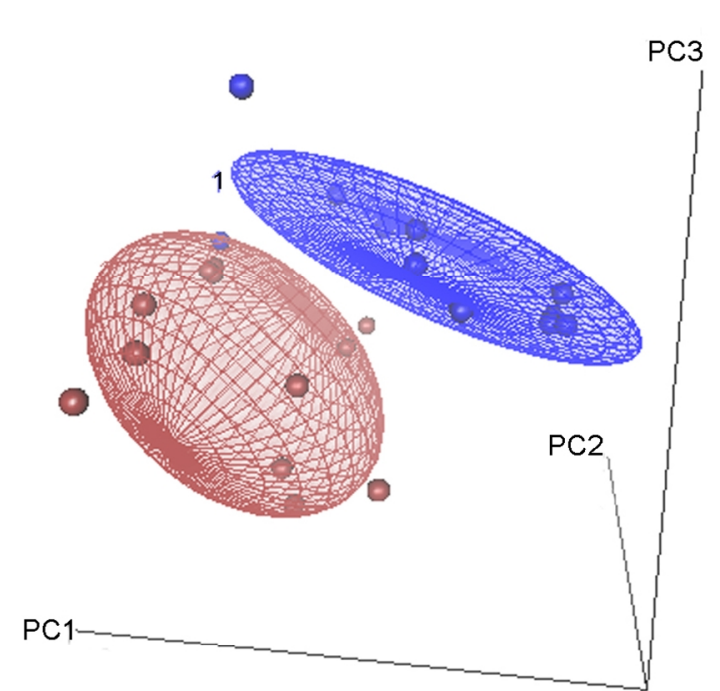

b

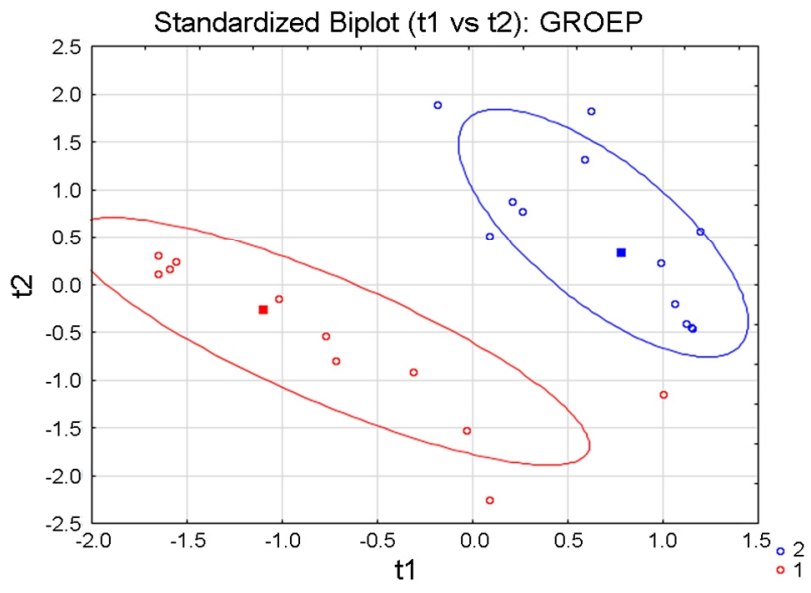

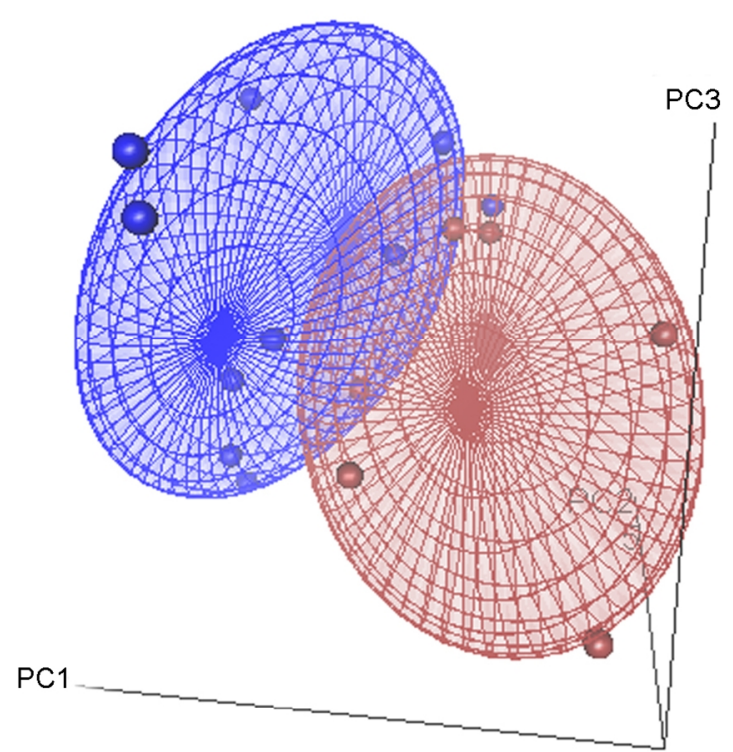

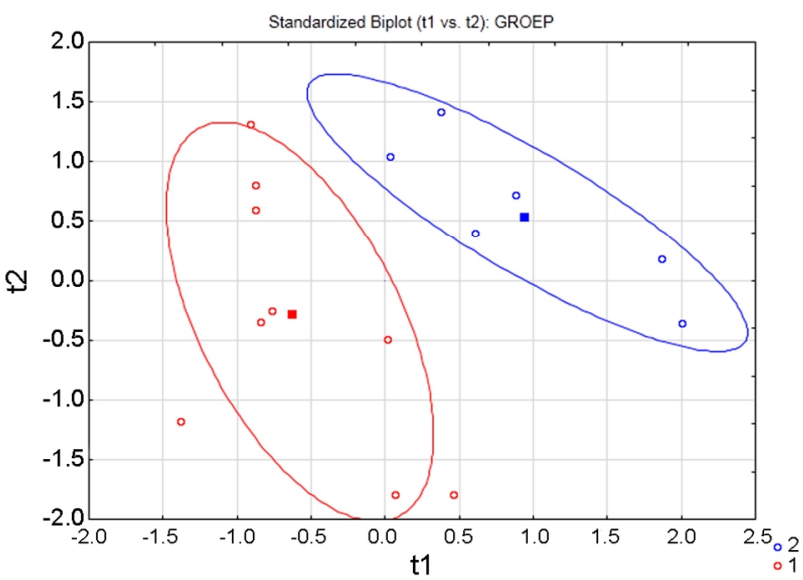

Fig. 5 Multivariate analyses of metabolite data from negative controls and HIV+ individuals are presented. a PCA score plots (unsupervised analysis) shown in 3-dimensions of different rotations to indicate the optimal view on the separation between the controls (shown in blue) and the infected individuals (shown in red). The figure to the left indicates complete separation of the cases from batch 1, but only partial separation for those from batch 2 . Three principal components were extracted for both bathes which explained $84 \%$ and $75 \%$ of the variation in the data for batch 1 and 2 respectively. b PLS-DA score plots (supervised analyses), indicates the separation as in a, using the same colour notation. Two components were extracted for both batches for which $72 \%$ (batch 1) and 50\% (batch 2) of the variation in the X-space and $87 \%$ (batch 1) and $80 \%$ (batch 2) of the variation in the Y-space were explained 


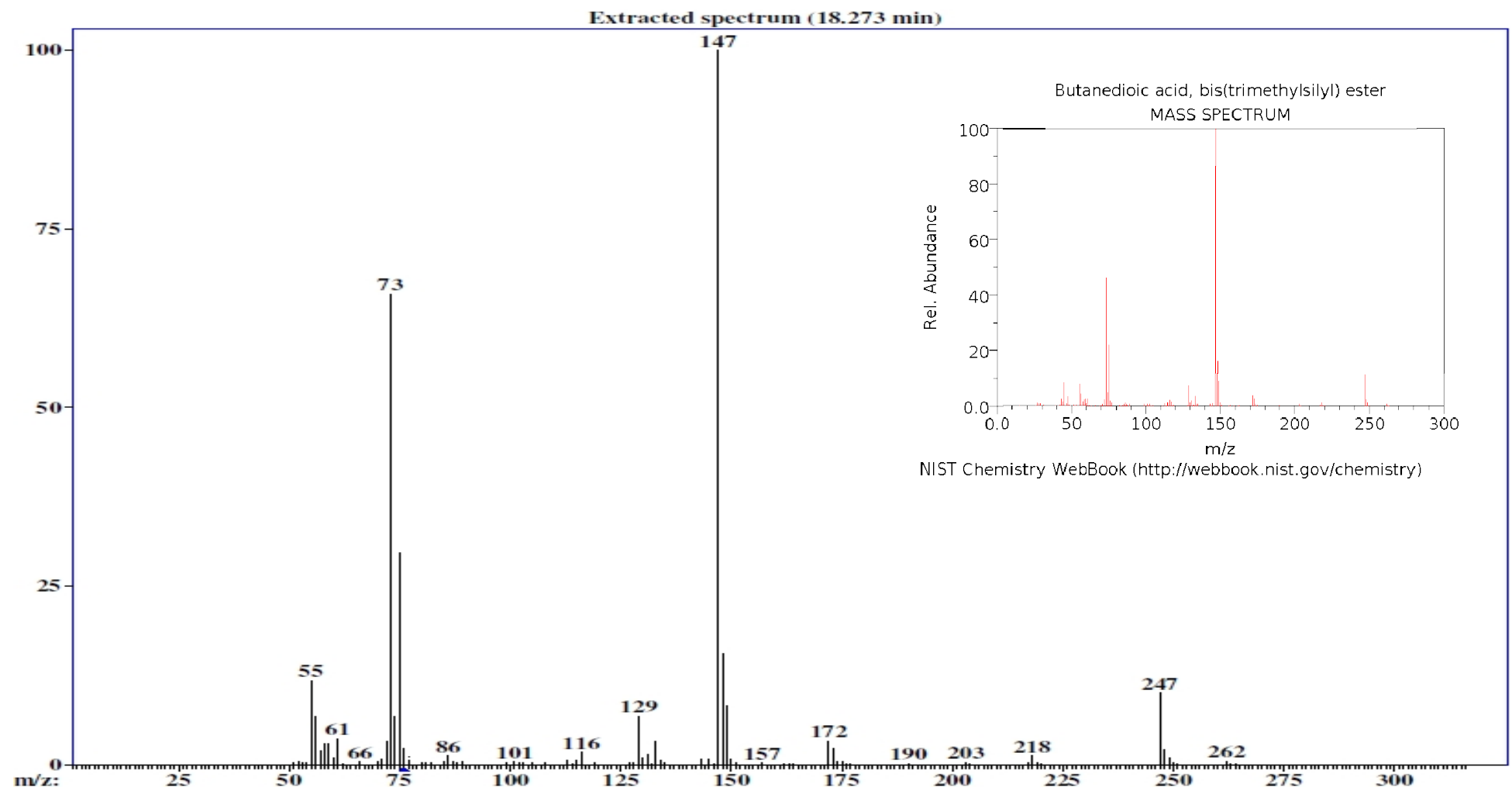




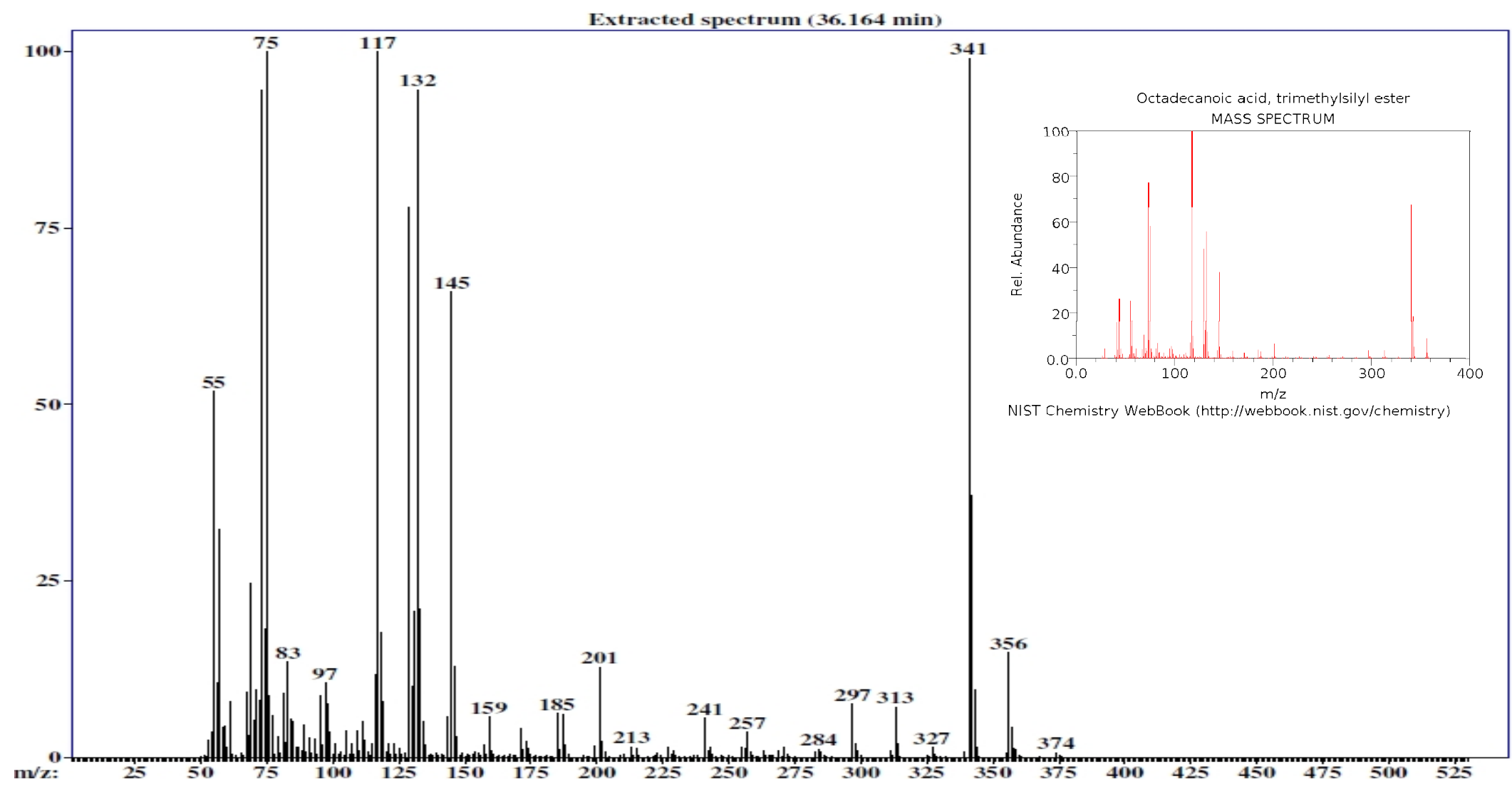

b

Fig. 6 Representative mass spectra of a) butanedioic acid, bis(trimethylsilyl) ester and b) octadecanoic acid, trimethylsilyl ester following derivatization, electron impact GCMS analysis, deconvolution and identification of the metabolites through the NIST 08 library. Representative spectra were taken from each of the batches 
- Markers of mitochondrial/metabolic dysfunction

At least four of the metabolites listed in Table 2 are generally regarded as markers of mitochondrial dysfunction. All four compounds were significantly higher in the HIV-infected individuals than in the controls. Of these organic acids, succinic acid and fumaric acid are intermediates in the second half of the Krebs cycle. Barshop (2004) noted increased urinary fumaric acid and malic acid in a metabolomics investigation of urine samples from patients with mitochondrial disorders. We have also recently reported (Reinecke et al. 2011) that succinic, fumaric and malic acid increased in the urine of patients suffering from a dysfunctional mitochondrial RC. The order of significance of elevated TCA metabolites in that study was found to be succinate $>$ fumarate $>$ malate. The perturbations in the metabolite profile were related to increased production of NADH $\left(\mathrm{NADH} / \mathrm{NAD}^{+}\right.$ratio) and $\mathrm{FADH}_{2}$ due to the dysfunctional RC. Impaired functioning of components of the RC has also been reported as a consequence of HIV infection (Ricci et al. 2004; Ladha et al. 2005).

Adipic and suberic acids, both products of fatty acid degradation, were significantly higher in HIV-infected individuals than in controls. Decreased electrotransfer flavoprotein (ETF) and increased ETF-ubiquinone oxidoreductase (QO), due to an impaired flow of electrons past $\mathrm{CoQ}_{10}$, may give rise to these two metabolites, which is known to occur during impaired mitochondrial fatty acid oxidation. Amongst other reactions, these metabolites are formed by omega $(\omega)$-oxidation of their respective short- and medium-chain fatty acid-CoA precursors, in accordance with impaired mitochondrial fatty acid oxidation (reviewed by Duran 2005). Adipic and suberic acids were likewise included as indicators, but not as specific markers, of complex I deficiency in patients with clinical defects of the RC (Reinecke et al. 2011). In general, therefore, it seems that the metabolic consequences of deficiencies in mitochondrial enzymes are reflected in the increased serum profile of these related markers in $\mathrm{HIV}+$ individuals, compared to controls.

- Saturated and unsaturated fatty acids, other lipids and metabolites involved in lipid metabolism

Alterations in lipid metabolism were noticed and described in the early phases of AIDS research. Hypertriglyceridemia was reported as early as 1989 (Grunfeld et al. 1989), and an increase in free fatty acids was reported to occur during the early stages of HIV infection (Grunfeld et al. 1992).

Six fatty acids were more prominent in treatment naive HIV+ individuals relative to controls in our study, as shown in Table 2. This was an important finding also in the global metabolomics investigation of SIV infection in rhesus macaques (Wikoff et al. 2008). The elevated concentration of free fatty acids, associated with increased expression of the PLA1A and PLA2G4C phospholipases in brain tissue of SIV-infected animals with 
Table 2. Summary of elevated metabolites identified as being indicators of HIV infection in this metabolomics study.

Features from MET-IDEA/NIST 08 Library

\begin{tabular}{l}
\hline Butanedioic acid, bis(trimethylsilyl) ester \\
2-Butenedioic acid (E)-, bis(trimethylsilyl) ester \\
Hexanedioic acid, bis(trimethylsilyl) ester
\end{tabular}

Hexanedioic acid, bis(trimethylsilyl) ester

Octanedioic acid, bis(trimethylsilyl) ester

\section{Common and alternative names}

\section{Indicators of mitochondrial/metabolic dysfunction}

Succinic acid / 1,4-Butanedioic acid

Fumaric acid / trans-1,2-Ethylenedicarboxylic acid

Adipic acid / 1,6-Hexanedioic acid

Suberic acid / 1,6-Hexanedicarboxylic acid

\section{Formula Batch ES (FC)}

t-test Mann-

Whitney

\begin{tabular}{|c|c|c|c|c|c|c|}
\hline \multicolumn{7}{|c|}{ Saturated and unsaturated fatty acids, other lipids and lipid metabolism } \\
\hline Decanoic acid, trimethylsilyl ester & Capric acid / Decoic acid & $\mathrm{C}_{10} \mathrm{H}_{20} \mathrm{O}_{2}$ & 1 & $2.49(3.92)$ & $<0.0001$ & $<0.0001$ \\
\hline Azelaic acid, bis(trimethylsilyl) ester & Azelaic acid / Nonanedioic acid & $\mathrm{C}_{9} \mathrm{H}_{16} \mathrm{O}_{4}$ & 1 & $1.72(5.30)$ & $<0.0001$ & 0.0001 \\
\hline Oleamide, N-trimethylsilyl- & Oleamide / Oleoylethanolamide & $\mathrm{C}_{18} \mathrm{H}_{35} \mathrm{NO}$ & 1 & $1.01(23.62)$ & 0.0074 & 0.0106 \\
\hline Octadecanoic acid, trimethylsilyl ester & Stearic Acid / Octadecanoic acid & $\mathrm{C}_{18} \mathrm{H}_{36} \mathrm{O}_{2}$ & 2 & $1.79(6.15)$ & 0.0003 & 0.0001 \\
\hline Arachidonic acid, trimethylsilyl ester & Arachidonic Acid / 5,8,11,14-Eicosatetraenoic acid & $\mathrm{C}_{20} \mathrm{H}_{32} \mathrm{O}_{2}$ & 2 & $1.28(3.80)$ & 0.0098 & 0.0001 \\
\hline 11-trans-Octadecenoic acid, trimethylsilyl ester & Vaccenic acid / 11-Octadecenoic acid & $\mathrm{C}_{18} \mathrm{H}_{34} \mathrm{O}_{2}$ & 2 & $0.88(61.84)$ & 0.0187 & 0.0312 \\
\hline \multicolumn{7}{|c|}{ Oxidative stress related metabolites } \\
\hline N,O-Bis-(trimethylsilyl)-2-pyrrolidone carboxylic acid & Pyroglutamic acid / 5-oxoproline & $\mathrm{C}_{5} \mathrm{H}_{7} \mathrm{NO}_{3}$ & 1 & $2.08(16.48)$ & $<0.0001$ & $<0.0001$ \\
\hline Cholest-5-en-7-one, 3-[(trimethylsilyl)oxy]- & 7-ketocholesterol / 3b-Hydroxycholest-5-en-7-one & $\mathrm{C}_{27} \mathrm{H}_{44} \mathrm{O}_{2}$ & 1 & $0.95(3.61)$ & 0.0136 & 0.0129 \\
\hline \multicolumn{7}{|c|}{ Other metabolites recognized as part of the human serum metabolome } \\
\hline $\begin{array}{l}\text { 1H-Indole-3-acetic acid, 1-(trimethylsilyl)-, trimethylsilyl } \\
\text { ester }\end{array}$ & Indole acetic acid / Indole-3-acetic acid & $\mathrm{C}_{10} \mathrm{H}_{9} \mathrm{NO}_{2}$ & 2 & $1.33(17.34)$ & 0.0131 & 0.0159 \\
\hline Benzaldehyde, 4-[(trimethylsilyl)oxy]- & 4-hydroxybenzaldehyde & $\mathrm{C}_{7} \mathrm{H}_{6} \mathrm{O}_{2}$ & 2 & $1.12(10.91)$ & 0.0345 & 0.0312 \\
\hline
\end{tabular}


encephalitis, was apparently linked to areas of infection and inflammation. We did not assess phospholipase expression or activity in the investigation reported here. It is, however, noteworthy that stearic acid (also reported by Wikoff et al. 2008), arachidonic acid and vaccenic acid are constituents of phospholipids. Arachidonic acid is a membrane glycerophospholipid, a substrate for phospholipase A2 (PLA2) and mediates inflammation. Vaccenic acid is a structural component of the cardiolipins (bisphosphatidyl glycerol), which are important components of the inner mitochondrial membrane, where they constitute about $20 \%$ of the total lipid content and abound in metabolically active heart and skeletal muscle cells.

Basic and applied research on the biological functions of oleamide (included in Table 2) was greatly stimulated by the discovery that it induces satiety in humans, reduced body weight in experimental animals (Roderiguez de Fonseca et al. 2001) and may present a pharmacological or nutritional means of addressing the growing prevalence of obesity in developed/rich societies. Oleamide is an endogenous substance and also an amide of the fatty acid oleic acid. It modulates lipid metabolism (Roderiguez de Fonseca et al. 2001) through its lipolytic properties that inhibit adipogenesis in adipose tissue (Matias et al. 2007) and by activation of $\beta$ oxidation in muscle (Guzman et al. 2004). The presence of oleamide increases markedly in treatment naive HIV+ individuals, who are also susceptible to wasting (Table 2, Grunfeld and Kotler 1992). Indeed, wasting was defined as early as 1992 by the Centres for Disease Control and Prevention as a diagnostic criterion for AIDS prior to the onset of opportunistic infections and in the absence of other, identifiable causes of weight loss. It is, however, premature to emphasize this observation prior to further verification.

Azelaic and capric acid are endogenous fatty acids of the human serum metabolome (Psychogios et al. 2011) but their presence may also be of exogenous origin. Candida albicans, a cause of opportunistic infections during HIV/AIDS is best treated by antibiotics which in the case of immune-compromised patients can be problematic (Pfaller and Dickema 2007) in that it promotes yeast infections. According to the Human Metabolome Database, azelaic acid is a new topical drug for treating hyperpigmentary disorders and is also known to treat opportunistic infections during HIV/AIDS, as is capric acid. Self-medication (rather than HIV infection) may therefore be a source of azelaic and capric acid.

\section{- Oxidative stress-related metabolites}

The increase in pyroglutamic acid in HIV+ individuals is statistically significant (Table 2). Two possible relationships between elevated pyroglutamic acid and HIV infection can be envisaged: (1) in experimental animals, increased pyroglutamine significantly inhibits mitochondrial complex I and III activities (Silva et al. 
2001), which might indicate that pyroglutamic acid is linked to HIV-induced mitochondrial dysfunction, as discussed above. (2) Pyroglutamic acid is a key intermediate in the $\gamma$-glutamyl cycle and is indicative of gluthatione and $\gamma$-glutamylcysteine synthetase deficiency (Jellum et al. 1970; Larsson et al. 1985). Oxidative stress is generally accepted as being involved in many aspects of HIV disease pathogenesis and could result in altered GSH/GSSG levels (glutathione/glutathione disulphides), a key factor in the defence against the devastating consequences of oxidative stress (Staal et al. 1992). That molecules relevant to oxidative stress would increase is confirmatory of the flow cytometry findings of Macho et al. (1995).

The measurement of oxysterols in plasma is reliable and a rapid means for assaying cholesterol oxidation products and oxidative stress/antioxidant balance (Iuliano et al. 2003). Nonenzymatic production of 7ketocholesterol, which was present among the most important metabolites associated with HIV infection (Table 2), was reported to arise via a free radical-mediated mechanism operating under conditions of oxidative stress (Bjorkhem and Diczfalusy 2002). In vitro, 7-ketocholesterol has wide-ranging potent effects and can induce apoptosis in vascular cells. The formation of 7-ketocholesterol by a mechanism involving free radicals concurs with its production from experimentally oxidized LDL. Whether this mechanism relates to previous observations of a marked difference in LDL between controls and ART-treated patients (Philippeos et al. 2009) requires further investigation.

- Other metabolites recognized as part of the human serum metabolome

The two other metabolites identified with HIV infection, indole acetic acid and 4-hydroxybenzaldehyde (Table 2) do not seem to be clearly linked to this pathological state. Indole acetic acid is a catabolic product of tryptophan metabolism (tryptophan levels usually decline during HIV infection). In humans it is mostly linked to the action of bacteria in the gut. Some indole acetic acid is also produced endogenously in mammalian tissues as the acid may be generated by the decarboxylation of tryptamine or the oxidative deamination of tryptophan. Likewise, 4-hydroxybenzaldehyde appears to be a human metabolite of less significance with regard to the pathology of HIV, although its presence was noted in the notorious metabolomics investigation of serum markers for prostate cancer (Sreekumar et al. 2009).

The CD4 count is currently regarded as one of the most important prognostic markers for HIV infection, even in the light of its many disadvantages. No appropriate correlation was done on the CD4 count and the important metabolites identified in this study but the categories of metabolites disclosed by this investigation proved to be sufficiently relevant and significant to be taken into account in studies on HIV infection. It can 
hardly be expected that a small number of metabolite biomarkers could account for all the cellular aberrations caused by HIV infection. This concurs with the divergent rather than convergent association between the metabolites presented in this paper (Table 2), and is in agreement with data reported by Wikoff et al. (2008) for SIV-infected rhesus macaques. It is, however, noteworthy that most metabolites indicative of mitochondrial dysfunction were present in the organic acids from batch 1, which had the highest values for the HIV-RNA in plasma. The higher apoptosis measured in HIV+ individuals is also in agreement with the degree of mitochondrial dysfunction measured through organic acid profiling. This may indicate that for the chronic phase of the disease the organic acid profile develops from mild organic acidemia in the early stages to more advanced organic acidemia, including markers of mitochondrial dysfunction in the later stages. Whether this is a consistent characteristic of disease progression - from chronic HIV infection to full-blown AIDS - will have to be addressed by more detailed investigations.

\section{Concluding Remarks}

A GC-MS investigation of the organic acid portion of the metabolome of human serum, linked to systematic bioinformatics analysis using univariate and multivariate statistical methods, resulted in the identification of 14 metabolites whose presence was significantly different between controls and HIV-infected individuals. At least 10 of these metabolites could be related to mitochondrial dysfunction, changes in lipid metabolism and oxidative stress, all of which are known aberrations of HIV infection. Mitochondrial dysfunction is a pathological consequence of HIV infection which was also confirmed through the detection of apoptosis. These observations were obtained by analysing HIV+ individuals who, according to their CD4 counts, were in the chronic, asymptomatic phase (WHO stage 2) of the disease. These individuals were not on antiretroviral treatment at the time their blood was collected. This study therefore indicated that a metabolomics investigation can disclose information on the markers that define the asymptomatic stage of HIV infection and may be developed into a method for monitoring more advanced stages of the disease and potentially also the response of infected individuals to ART.

\section{Acknowledgements}

This work was supported by the following institutions and agencies: the National Research Foundation; Biotechnology Partnerships and Development (BIOPAD, now called the Technology Innovation Agency); the 
Medical Research Council; the Centre for the Study of AIDS and the Faculty of Science at the University of Pretoria.

\section{Conflict of Interest}

The authors declare no conflict of interest.

\section{References}

Barker, M. and Rayens, W. (2003). Partial least squares for discrimination. J Chemometrics. 17, 166-173.

Banda, N.K., Bernier, J., Kurahara, D.K. et al. (1992). Crosslinking CD4 by human immunodeficiency virus gp120 primes T cells for activation-induced apoptosis. J Exp Med. 176, 1099-1106.

Barshop, B.A. (2004). Metabolomic approaches to mitochondrial disease: correlation of urine organic acids. Mitochondrion. 4, 521-527.

Bjorkhem, I. and Diczfalusy, U. (2002). Oxysterols: friends, foes, or just fellow passengers? Arterioscler Thromb Vasc Biol. 22, 734-742.

Broeckling, C.D., Reddy, I.R., Duran, A.L., Zhao, X. and Sumner, L.W. (2006). MET-IDEA: data extraction tool for mass spectrometry-based metabolomics. Anal Chem. 78, 4334-4341.

Calabrese, L.H., Proffitt, M.R., Gupta, M.K. et al. (1984). Serum beta 2-microglobulin and interferon in homosexual males: relationship to clinical findings and serologic status to the human $\mathrm{T}$ lymphotropic virus (HTLV-III). AIDS Res. 1, 423-438.

Calza, L., Manfredi, R. and Chiodo, F. (2003). Hyperlipidaemia in patients with HIV-1 infection receiving highly active antiretroviral therapy: epidemiology, pathogenesis, clinical course and management. Int J Antimicrob Agents. 22, 89-99.

Centlivre, M., Sala, M., Wain-Hobson, S. and Berkhout, B. (2007). In HIV-1 pathogenesis the die is cast during primary infection. AIDS. 21, 1-11. 
Dunn, W.B., Broadhurst, D., Begley, P. et al. (2011). Procedures for large-scale metabolic profiling of serum and plasma using gas chromatography and liquid chromatography coupled to mass spectrometry. Nat Protoc. 6 , 1060-1083.

Duran, M. (2005). Disorders of mitochondrial fatty acid oxidation in Blau, N., Duran, M. and Blaskovics, M.E. (Eds), Physician's guide to the laboratory diagnosis of metabolic diseases. Revised $2^{\text {nd }}$ ed. Springer-Verlag, Heidelberg, pp. 309-334.

Gkrania-Klotsas, E. and Klotsas, A. (2007). HIV and HIV treatment: effects on fats, glucose and lipids. Br Med Bull. 84, 49-68.

Grunfeld, C., Kotler, D.P., Hamadeh, R., Tierney, A., Wang, J. and Pierson, R.N. (1989). Hypertriglyceridemia in the acquired immunodeficiency syndrome. Am J Med. 86, 27-31.

Grunfeld, C., Pang, M., Doerrler, W., Shigenaga, J.K., Jensen, P. and Feingold, K.R. (1992). Lipids, lipoproteins, triglyceride clearance, and cytokines in human immunodeficiency virus infection and the acquired immunodeficiency syndrome. J Clin Endocrinol Metab. 74, 1045-1052.

Grunfeld, C. and Kotler, D.P. (1992). Wasting in the acquired immunodeficiency syndrome. Semin Liver Dis. 12, 175-187.

Guzmán, M., Lo Verme, J., Fu, J., Oveisi, F., Blázquez, C. and Piomelli, D. (2004). Oleoylethanolamide Stimulates Lipolysis by Activating the Nuclear Receptor Peroxisome Proliferator-activated Receptor $\alpha$ (PPARa). J Biol Chem. 279, 27849-27854.

Hattingh, Z., Walsh, C., Veldman, F.J. and Bester,C.J. (2009). Metabolic profile of HIV-Infected Women in Mangaung, Free State. S Afr J Clin Nutr. 22, 23-28.

Hewer, R., Vorster, J., Steffens, F.E. and Meyer, D. (2006). Applying biofluid 1H NMR-based metabonomic techniques to distinguish between HIV-1 positive/AIDS patients on antiretroviral treatment and HIV-1 negative individuals. J Pharm Biomed Anal. 41, 1442-1446. 
Hoffman, G.F. and Feyh, P. (2005). Organic acid analysis in Blau, N., Duran, M. and Blaskovics, M.E. (Eds), Physician's guide to the laboratory diagnosis of metabolic diseases. Revised $2^{\text {nd }}$ ed. Springer-Verlag, Heidelberg, pp. 27-44.

Hommes, M.J.T., Romijn, J.A., Godfried, M.H. et al. (1990). Increased resting energy expenditure in human immunodeficiency virus-infected men. Metab Clin Exp. 39, 1186-1190.

Iuliano, L., Micheletta, F., Natoli, S. et al. (2003). Measurement of oxysterols and alpha-tocopherol in plasma and tissue samples as indices of oxidant stress status. Anal Biochem. 312, 217-223

Jansen, C.A., van Baarle, D. and Miedema, F. (2006). HIV-specific CD4+ T cells and viremia: who's in control? Trends Immunol. 27, 119-124.

Jellum, E., Kluge, T., Borresen, H.C., Stokke, O. and Eldjarn, L. (1970). Pyroglutamic aciduria--a new inborn error of metabolism. Scand J Clin Lab Invest. 26, 327-335.

Kanekar, A. (2010). Biomarkers predicting progression of human immunodeficiency virus-related disease. J Clin Med Res. 2, 55-61.

Krylov, S.N., Arriaga, E.A., Chan, N.W., Dovichi, N.J. and Palcic, M.M. (2000). Metabolic cytometry: monitoring oligosaccharide biosynthesis in single cells by capillary electrophoresis. Anal Biochem. 283, 133135.

Ladha, J.S., Tripathy, M.K. and Mitra, D. (2005). Mitochondrial complex I activity is impaired during HIV-1induced T-cell apoptosis. Cell Death Differ. 12, 1417-1428.

Larsson, A., Wachtmeister, L., von Wendt, L., Andersson, R., Hagenfeldt, L. and Herrin, K.M. (1985). Ophthalmological, psychometric and therapeutic investigations in two sisters with hereditary glutathione synthetase deficiency (5-oxoprolinuria). Neuropediatrics. 16, 131-136.

Macho, A., Castedo, M., Marchetti, P. et al. (1995). Mitochondrial dysfunctions in circulating T lymphocytes from human immunodeficiency virus-1 carriers. Blood. 86, 2481-2487. 
Matias, I., Gonthier, M.P., Petrosino, S. et al. (2007). Role and regulation of acylethanolamides in energy balance: focus on adipocytes and beta-cells. Br J Pharmacol. 152, 676-690.

Meyaard, L., Otto, S., Jonker, R., Mijnster, M., Keet, R. and Miedema, F. (1992). Programmed death of T cells in HIV-1 infection. Science. 257, 217-219.

Nakagawa, S. and Cuthill, I.C. (2007). Effect size, confidence interval and statistical significance: a practical guide for biologists. Biol Rev Camb Philos Soc. 82, 591-605.

Nordström, A. and Lewensohn, R. (2010). Metabolomics: moving to the clinic. J Neuroimmune Pharmacol. 5, 4-17.

Pendyala, G. and Fox, H.S. (2010). Proteomic and metabolomic strategies to investigate HIV-associated neurocognitive disorders. Genome Med. 2, 22.

Pfaller, M.A. and Diekema, D.J. (2007). Epidemiology of invasive candidiasis: a persistent public health problem. Clin Microbiol Rev. 20, 133-163.

Philippeos, C., Steffens, F.E. and Meyer, D. (2009). Comparative 1H NMR-based metabonomic analysis of HIV-1 sera. J Biomol NMR. 44, 127-137.

Pinti, M., Nasi, M., Gibellini, L. et al. (2010). The Role of Mitochondria in HIV Infection and Its Treatment. J Exp Clin Med. 2, 145-155.

Pitt, J.J., Eggington, M. and Kahler, S.G. (2002). Comprehensive screening of urine samples for inborn errors of metabolism by electrospray tandem mass spectrometry. Clin Chem. 48, 1970-1980.

Psychogios, N., Hau, D.D., Peng, J. et al. (2011). The Human Serum Metabolome. PLoS ONE. 6, e16957.

Redestig, H., Fukushima, A., Stenlund, H. et al. (2009). Compensation for systematic cross-contribution improves normalization of mass spectrometry based metabolomics data. Anal Chem. 81, 7974-7980.

Reinecke, C., Koekemoer, G., van der Westhuizen, F.H. et al. (2011). Metabolomics of urinary organic acids in respiratory chain deficiencies in children. Metabolomics. 1-20. 
Ricci, J.E., Munoz-Pinedo, C., Fitzgerald, P. et al. (2004). Disruption of mitochondrial function during apoptosis is mediated by caspase cleavage of the p75 subunit of complex I of the electron transport chain. Cell. $117,773-786$.

Rodriguez de Fonseca, F., Navarro, M., Gomez, R. et al. (2001). An anorexic lipid mediator regulated by feeding. Nature. 414, 209-212.

Roederer, M. (2001). Spectral compensation for flow cytometry: visualization artifacts, limitations, and caveats. Cytometry. 45, 194-205.

Roshal, M., Zhu, Y. and Planelles, V. (2001). Apoptosis in AIDS. Apoptosis. 6, 103-116.

Silva, A.R., Silva, C.G., Ruschel, C. et al. (2001). L-pyroglutamic acid inhibits energy production and lipid synthesis in cerebral cortex of young rats in vitro. Neurochem Res. 26, 1277-1283.

Sreekumar, A., Poisson, L.M., Rajendiran, T.M. et al. (2009). Metabolomic profiles delineate potential role for sarcosine in prostate cancer progression. Nature. 457, 910-914.

Staal, F.J.T., Ela, S.W., Roederer, M., Anderson, M.T., Herzenberg, L.A. and Herzenberg, L.A. (1992). Glutathione deficiency and human immunodeficiency virus infection. Lancet. 339, 909-912.

Sysi-Aho, M., Katajamaa, M., Yetukuri, L. and Oresic, M. (2007). Normalization method for metabolomics data using optimal selection of multiple internal standards. BMC Bioinformatics. 8, 93.

Touloumi, G. and Hatzakis, A. (2000). Natural history of HIV-1 infection. Clin Dermatol. 18, 389-399.

Trygg, J., Holmes, E. and Lundstedt, T. (2007). Chemometrics in metabonomics. J Proteome Res. 6, 469-479.

Tuck, M.K., Chan, D.W., Chia, D. et al. (2009). Standard operating procedures for serum and plasma collection: early detection research network consensus statement standard operating procedure integration working group. J Proteome Res. 8, 113-117.

Tusher, V.G., Tibshirani, R. and Chu, G. (2001). Significance analysis of microarrays applied to the ionizing radiation response. Proc Natl Acad Sci. 98, 5116-5121. 
UNAIDS. (2002). AIDS Epidemic Update, Geneva, Switzerland. http://data.unaids.org/publications/IRCpub03/epiupdate2002 en.pdf. Accessed 5 July 2011.

UNAIDS. (2009). AIDS Epidemic Update, Geneva, Switzerland. http://data.unaids.org/pub/report/2009/jc1700_epi_update_2009_en.pdf. Accessed 5 July 2011.

van Engeland, M., Nieland, L.J., Ramaekers, F.C., Schutte, B. and Reutelingsperger, C.P. (1998). Annexin Vaffinity assay: a review on an apoptosis detection system based on phosphatidylserine exposure. Cytometry. 31, $1-9$.

Verde, V., Fogliano, V., Ritieni, A., Maiani, G., Morisco, F. and Caporaso, N. (2002). Use of N,N-dimethyl-pphenylenediamine to evaluate the oxidative status of human plasma. Free Radic Res. 36, 869-873.

Whitmore, C.D., Hindsgaul, O., Palcic, M.M., Schnaar, R.L. and Dovichi, N.J. (2007). Metabolic cytometry. Glycosphingolipid metabolism in single cells. Anal Chem. 79, 5139-5142.

Wikoff, W.R., Pendyala, G., Siuzdak, G. and Fox, H.S. (2008). Metabolomic analysis of the cerebrospinal fluid reveals changes in phospholipase expression in the CNS of SIV-infected macaques. J Clin Invest. 118, 26612669.

Wohl, D.A., McComsey, G., Tebas, P. et al. (2006). Current Concepts in the Diagnosis and Management of Metabolic Complications of HIV Infection and Its Therapy. Clin Infect Dis. 43, 645-653.

World Health Organization. (2005). Interim WHO clinical staging of HIV/AIDS and HIV/AIDS case definitions for surveillance - Africa region. http://www.who.int/hiv/pub/guidelines/clinicalstaging/pdf. Accessed 1 July 2011.

World health Organization. (2006). Antiretroviral therapy for HIV infection in adults and adolescents: Recommendations for a public health approach. http://www.who.int/hiv/pub/guidelines/artadultguidelines.pdf, p.12. Accessed 1 July 2011.

Xu, F., Zou, L. and Ong, C.N. (2009). Multiorigination of chromatographic peaks in derivatized GC/MS metabolomics: a confounder that influences metabolic pathway interpretation. J Proteome Res. 8, 5657-5665. 